\title{
An approximation method for the stabilizing solution of the Hamilton-Jacobi equation for integrable systems using Hamiltonian perturbation theory
}

\author{
Noboru Sakamoto and Arjan J. van der Schaft
}

\begin{abstract}
In this report, a method for approximating the stabilizing solution of the Hamilton-Jacobi equation for integrable systems is proposed using symplectic geometry and a Hamiltonian perturbation technique. Using the fact that the Hamiltonian lifted system of an integrable system is also integrable, the Hamiltonian system (canonical equation) that is derived from the theory of 1-st order partial differential equations is considered as an integrable Hamiltonian system with a perturbation caused by control. Assuming that the approximating Riccati equation from the Hamilton-Jacobi equation at the origin has a stabilizing solution, we construct approximating behaviors of the Hamiltonian flows on a stable Lagrangian submanifold, from which an approximation to the stabilizing solution is obtained.
\end{abstract}

\section{INTRODUCTION}

When analyzing a control system or designing a feedback control, one often encounters certain types of equations that dominate fundamental properties of the control problem at hand. It is the Riccati equation for linear systems and the Hamilton-Jacobi equation plays the same role in nonlinear systems. For example, an optimal feedback control can be derived from a solution of a Hamilton-Jacobi equation [15] and $H^{\infty}$ feedback controls are obtained by solving one or two Hamilton-Jacobi equations [3], [14], [21], [22]. Closely related to optimal control and $H^{\infty}$ control is the notion of dissipativity, which is also characterized by a HamiltonJacobi equation (see, e.g., [13], [24]). Some active areas of research in recent years are the factorization problem [4], [5] and the balanced realization problem [11] and the solutions of these problems are again represented by Hamilton-Jacobi equations (or, inequalities).

Contrary to the well-developed theory and computational tools for the Riccati equation, which are widely applied, the Hamilton-Jacobi equation is still an impediment to practical applications of nonlinear control theory. There seem to be mainly two directions in the study of the Hamilton-Jacobi equation. One is to try to obtain approximate solutions and the other is to study the geometric structure and the properties of the equation itself and its exact solutions. For the former research we refer to [16] for the Taylor series expansion approach, [7] for the Galerkin approximation method, and [17] for the state dependent Riccati equation approach.

N. Sakamoto is with the Department of Aerospace Engineering, Nagoya University, Furo-cho, Chikusa-ku, Nagoya, 464-8603, Japan sakamoto@nuae.nagoya-u.ac.jp

A. J. van der Schaft is with Institute for Mathematics and Computing Science, University of Groningen, 9700 AV Groningen, The Netherlands A.J.van.der. Schaft@math.rug.nl
A partially related research field to the former direction is the theory of viscosity solutions. It deals with general HamiltonJacobi equations for which classical (differentiable) solutions do not exist. For introductions to viscosity solutions see, for instance, [6], [8], [10] and for an application to an $H^{\infty}$ control problem, see [20]. In the second direction, [21] and [22] give a sufficient condition for the existence of the stabilizing solution using symplectic geometry. In [19], the geometric structure of the Hamilton-Jacobi equation is studied showing the similarity and difference with the Riccati equation. See also [23] for the treatment of the HamiltonJacobi equation as well as recently developed techniques in nonlinear control theory such as the theory of portHamiltonian systems.

In this report, we attempt to develop a method to approximate the stabilizing solution of the Hamilton-Jacobi equation based on the geometric research in [21], [22] and [19]. The main object of the geometric research on the Hamilton-Jacobi equation is the associated Hamiltonian system. However, most approximation research papers mentioned above do not explicitly consider Hamiltonian systems, although it is well-known that the Hamiltonian matrix plays a crucial role in the calculation of the stabilizing solution for the Riccati equation. One of our purposes in this report is to fill in this gap. Another purpose is to explore the possibility of using integrability conditions on the uncontrolled part of the system for controller design. Even when one can completely solve the equations of motion for a system with zero input, most nonlinear control techniques do not exploit the knowledge because once a feedback control is implemented, the system is not integrable anymore. However, within the geometric framework for the Hamilton-Jacobi equation, the effect of control can be considered as a Hamiltonian perturbation to the Hamiltonian system obtained by lifting the original equations of motion. Here, a crucial property is that if the equations of motion are integrable, then its lifted Hamiltonian system is also integrable (see, §IV). By using one of the Hamiltonian perturbation techniques (see, e.g., [2], [12]) we analyze the behaviors of the Hamiltonian systems with control effects and try to approximate the Lagrangian submanifold on which the Hamiltonian flow is asymptotically stable.

The organization of this report is as follows. In $\S \mathrm{II}$, the theory of 1st-order partial differential equations is reviewed in the framework of symplectic geometry, stressing the one-to-one correspondence between solution and Lagrangian 
submanifold. In $\S$ III, a special type of solution, called the stabilizing solution, is introduced and the geometric theory for the Riccati equation is also reviewed. In $\S I V$ a key observation on integrability for Hamiltonian lifted systems is presented. We apply, in $\S \mathrm{VI}$, a Hamiltonian perturbation technique (reviewed in $\S \mathrm{V}$ ) for the system in which the Hamiltonian is decomposed into an integrable one and a perturbation Hamiltonian that is related to influence of control. By assuming that the linearized Riccati equation at the origin has a stabilizing solution, we try to approximate the behaviors on the stable Lagrangian submanifold. Once the flows approximating the actual asymptotically stable Hamiltonian flows are obtained with general initial conditions, it is an algebraic operation to obtain the surface on which these flows are moving. In $\S$ VII, a numerical example is illustrated. For the sake of space, we have omitted proofs for propositions.

\section{REVIEW OF THE THEORY OF 1ST-ORDER PARTIAL DIFFERENTIAL EQUATIONS}

In this section we outline, by using the symplectic geometric machinery, the essential parts of the theory of partial differential equations of first order.

Let us consider a partial differential equation of the form

$$
F\left(x_{1}, \cdots, x_{n}, p_{1}, \cdots, p_{n}\right)=0,
$$

where $F$ is a $C^{\infty}$ function of $2 n$ variables, $x_{1}, \cdots, x_{n}$ are independent variables, $z$ is an unknown function and $p_{1}=\partial z / \partial x_{1}, \cdots, p_{n}=\partial z / \partial x_{n}$. Let $M$ be an $n$ dimensional space for $\left(x_{1}, \cdots, x_{n}\right)$. We regard the $2 n$ dimensional space for $(x, p)=\left(x_{1}, \cdots, x_{n}, p_{1}, \cdots, p_{n}\right)$ as the cotangent bundle $T^{*} M$ of $M . T^{*} M$ is a symplectic manifold with symplectic form $\theta=\sum_{i=1}^{n} d x_{i} \wedge d p_{i}$.

Let $\pi: T^{*} M \rightarrow M$ be the natural projection and $V \subset T^{*} M$ be a hypersurface defined by $F=0$. Define a submanifold

$$
\Lambda_{Z}=\left\{(x, p) \in T^{*} M \mid p_{i}=\partial z / \partial x_{i}(x), i=1, \cdots, n\right\}
$$

for a smooth function $z(x)$. Then, $z(x)$ is a solution of (PD) if and only if $\Lambda_{Z} \subset V$. Furthermore, $\left.\pi\right|_{\Lambda_{Z}}: \Lambda_{Z} \rightarrow M$ is a diffeomorphism and $\Lambda_{Z}$ is a Lagrangian submanifold because $\operatorname{dim} \Lambda_{Z}=n$ and

$$
\left.\theta\right|_{\Lambda_{Z}}=0
$$

Conversely, it is well-known (see, e.g. [1], [18]) that for a Lagrangian submanifold $\Lambda$ passing through $q \in T^{*} M$ on which $\left.\pi\right|_{\Lambda}: \Lambda \rightarrow M$ is a diffeomorphism, there exists a neighborhood $U$ of $q$ and a function $z(x)$ defined on $\pi(U)$ such that

$$
\Lambda \cap U=\left\{(x, p) \in U \mid p_{i}=\partial z / \partial x_{i}(x), i=1, \cdots, n\right\} .
$$

Therefore, finding a solution of (PD) is equivalent to finding a Lagrangian submanifold $\Lambda \subset V$ on which $\left.\pi\right|_{\Lambda}: \Lambda \rightarrow M$ is a diffeomorphism.

Let $f_{1}=F$. To construct such a Lagrangian submanifold passing through $q \in T^{*} M$, and hence to obtain a solution defined on a neighborhood of $\pi(q)$, it suffices to find functions $f_{2}, \cdots, f_{n} \in \mathscr{F}\left(T^{*} M\right)$ with $d f_{1}(q) \wedge \cdots \wedge d f_{n}(q) \neq 0$ such that $\left\{f_{i}, f_{j}\right\}=0(i, j=1, \cdots, n)$, where $\{\cdot, \cdot\}$ is the Poisson bracket, and

$$
\left|\frac{\partial\left(f_{1}, \cdots, f_{n}\right)}{\partial\left(p_{1}, \cdots, p_{n}\right)}\right|(q) \neq 0 .
$$

Using these functions, equations $f_{1}=0, f_{j}=$ constant, $j=$ $2, \ldots, n$ define a Lagrangian submanifold $\Lambda \subset V$. Note that the condition (1) implies, by the implicit function theorem, that $\left.\pi\right|_{\Lambda}$ is a diffeomorphism on some neighborhood of $q$.

Since $\{F, \cdot\}$ is the Hamiltonian vector field $X_{F}$ with Hamiltonian $F$, the functions $f_{2}, \cdots, f_{n}$ above are first integrals of $X_{F}$. The ordinary differential equation that gives the integral curve of $X_{F}$ is the Hamilton's canonical equations

$$
\left\{\begin{array}{l}
\frac{d x_{i}}{d t}=\frac{\partial F}{\partial p_{i}} \\
\frac{d p_{i}}{d t}=-\frac{\partial F}{\partial x_{i}}
\end{array} \quad(i=1, \cdots, n),\right.
$$

and therefore, we seek $n-1$ commuting first integrals of (2) satisfying (1).

\section{THE STABILIZING SOLUTION OF THE HAMILTON-JACOBI EQUATION}

Let us consider the Hamilton-Jacobi equation often encountered in nonlinear control theory

$$
H(x, p)=p^{T} f(x)-\frac{1}{2} p^{T} R(x) p+q(x)=0,
$$

where $f: M \rightarrow \mathbb{R}^{n}, R: M \rightarrow \mathbb{R}^{n \times n}, q: M \rightarrow \mathbb{R}$ are all $C^{\infty}$, and $R(x)$ is a symmetric matrix for all $x \in M$. We also assume that $f$ and $q$ satisfy $f(0)=0, q(0)=0$ and $\frac{\partial q}{\partial x}(0)=0$.

The stabilizing solution of (HJ) is defined as follows.

Definition 1: A solution $z(x)$ of (HJ) is said to be the stabilizing solution if $p(0)=0$ and 0 is an asymptotically stable equilibrium of the vector field $f(x)-R(x) p(x)$, where $p(x)=(\partial z / \partial x)^{T}(x)$.

It will be important to understand the notion of the stabilizing solution in the framework of symplectic geometry described in the previous section. Suppose that we have the stabilizing solution $z(x)$ around the origin. Then, the Lagrangian submanifold corresponding to $z(x)$ is

$$
\Lambda_{Z}=\{(x, p) \mid p=\partial z / \partial x(x)\} \subset T^{*} M .
$$

$\Lambda_{Z}$ is invariant under the Hamiltonian flow of

$$
\left\{\begin{array}{l}
\dot{x}=f(x)-R(x) p \\
\dot{p}=-\frac{\partial f}{\partial x}(x)^{T} p+\frac{\partial\left(p^{T} R(x) p\right)^{T}}{\partial x}-{\frac{\partial q^{T}}{\partial x}}^{T}
\end{array}\right.
$$

To see this invariance, one needs to show that the second equation identically holds on $\Lambda_{Z}$, which can be done by taking the derivative of (HJ) after replacing $p$ with $p(x)$. Note that the left-hand side in the second equation of (3) restricted to $\Lambda_{Z}$ is $(\partial p / \partial x)(f(x)-R(x) p(x))$. The first equation is exactly the vector field in Definition 1 . Therefore, the 
stabilizing solution is the Lagrangian submanifold on which $\pi$ is a diffeomorphism and the Hamiltonian flow associated with $H(x, p)$ is asymptotically stable.

\section{AN OBSERVATION ON INTEGRABLE SYSTEMS AND THEIR HAMILTONIAN LIFTING}

It is well-known that any system described by an ordinary differential equation can be represented as a Hamiltonian system by doubling the system dimension (Hamiltonian lifting). In [9] this technique is extended to control systems with inputs and outputs and is known to be effective for fundamental control problems such as factorization [5], [4] and model reduction problems [11]. In this section we give a useful observation on a Hamiltonian lifted system when the original system is integrable. Although it is simple, we did not find this observation in the literature.

Let the system $\dot{x}=f(x)$ be completely integrable and $u_{1}(x), \ldots, u_{n-1}(x)$ be first integrals. Consider its Hamiltonian lifted system

$$
\left\{\begin{array}{l}
\dot{x}=\frac{\partial H}{\partial p}=f(x) \\
\dot{p}=-\frac{\partial H}{\partial x}=-\frac{\partial f}{\partial x}(x)^{T} p
\end{array}\right.
$$

with Hamiltonian $H=p^{T} f(x)$. Let $v_{j}(x, p)=u_{j}(x)$ for $j=1, \ldots, n-1$ and $v_{n}=H(x, p)$. Then,

$$
\begin{aligned}
& \left\{v_{i}, v_{j}\right\}=\frac{\partial v_{i}}{\partial x}{\frac{\partial v_{j}}{\partial p}}^{T}-\frac{\partial v_{i}}{\partial p}{\frac{\partial v_{j}}{\partial x}}^{T}=0 \\
& \quad \text { for } i, j=1, \ldots, n-1, \\
& \left\{v_{i}, v_{n}\right\}=\frac{\partial v_{i}}{\partial x} f(x)-\frac{\partial v_{i}}{\partial p} \frac{\partial\left(p^{T} f(x)\right)^{T}}{\partial x}=0 \\
& \quad \text { for } i=1, \ldots, n-1,
\end{aligned}
$$

which means that $v_{1}, \ldots, v_{n}$ are in involution. Therefore, the Hamiltonian system (4) is integrable in the sense of Liouville. This means that if one can obtain general solutions of the original system by quadrature, it is also possible for its lifted system.

One may realize that in the analysis of the Hamilton-Jacobi equation (HJ) Hamilton's canonical equations (3) contain the same terms as the Hamiltonian lifting (4) of the plant system. The purpose of this report is to show that one can exploit this property of Hamiltonian lifting for approximation of the stabilizing solution of $(\mathrm{HJ})$.

\section{THE VARIATION OF CONSTANTS TECHNIQUE IN HAMILTONIAN PERTURBATION THEORY}

We review, in this section, one of the Hamiltonian perturbation techniques which is a simple consequence of the Hamilton-Jacobi theory (see, e.g., [2], [12]).

Let

$$
H(x, p)=H_{0}(x, p)+H_{1}(x, p)
$$

be the Hamiltonian with the integrable part $H_{0}$ and the perturbation $H_{1}$. By the integrability condition, the HamiltonJacobi equation

$$
H_{0}\left(x, \frac{\partial S}{\partial x}\right)+\frac{\partial S}{\partial t}=0
$$

has a complete solution $S\left(x_{1}, \ldots, x_{n}, t, P_{1}, \ldots, P_{n}\right)$, where $P_{1}, \ldots, P_{n}$ are arbitrary constants. By the canonical coordinate transformation $(x, p) \rightarrow(X, P)$ defined by

$$
p_{j}=\frac{\partial S}{\partial x_{j}}, \quad X_{j}=\frac{\partial S}{\partial P_{j}}
$$

the new Hamiltonian becomes 0 and the unperturbed equations of motion

$$
\dot{x}_{j}=\frac{\partial H_{0}}{\partial p_{j}}, \quad \dot{p}_{j}=-\frac{\partial H_{0}}{\partial x_{j}}
$$

are converted into

$$
\dot{X}_{j}=0, \quad \dot{P}_{j}=0 .
$$

By the canonical transformation (6), the new Hamiltonian for the perturbed equations of motion is $H_{1}(x, p)$ since by (5) $S$ satisfies

$$
H\left(x, \frac{\partial S}{\partial x}\right)+\frac{\partial S}{\partial t}=H_{1}(x, p) .
$$

Therefore,

$$
\dot{x}_{j}=\frac{\partial H}{\partial p_{j}}, \quad \dot{p}_{j}=-\frac{\partial H}{\partial x_{j}}
$$

are converted into

$$
\dot{X}_{j}=\frac{\partial H_{1}}{\partial P_{j}}(x, p) \quad \dot{P}_{j}=-\frac{\partial H_{1}}{\partial X_{j}}(x, p),
$$

where, from (6), $x_{j}=x_{j}(t, X, P)$ and $p_{j}=p_{j}(t, X, P)$.

\section{CONTROL PROBLEM FOR INTEGRABLE SYSTEMS AND APPROXIMATION OF THE STABILIZING SOLUTION}

In this report we consider the nonlinear control system with $m$ inputs of the form

$$
\dot{x}=f(x)+G(x) u,
$$

where $f: \mathbb{R}^{n} \rightarrow \mathbb{R}^{n}$ and $G: \mathbb{R}^{n} \rightarrow \mathbb{R}^{n \times m}$ are smooth functions of $x$.

Assumption 1: The system under control $\dot{x}=f(x)$ is integrable and a solution $x=\Phi\left(t, x_{0}\right)$ for a general initial condition $x=x_{0}$ at $t=0$ is obtained by quadrature.

We consider the same Hamilton-Jacobi equation in $\S$ III

$$
H=p^{T} f(x)-\frac{1}{2} p^{T} R(x) p+q(x)=0
$$

where $q=\frac{1}{2} x^{T} Q x+O\left(|x|^{3}\right)$ with $Q$ a real constant $n \times n$ symmetric matrix. Being $R(x)$ and $q(x)$ positive semidefinite in $(\mathrm{HJ})$ corresponds to an optimal control problem whereas $R(x)$ and $q(x)$ are indefinite for an $H^{\infty}$ type problem.

The Hamilton's canonical equations to solve $(\mathrm{HJ})$ are

$$
\left\{\begin{array}{l}
\dot{x}=f(x)-R(x) p \\
\dot{p}=-\frac{\partial f}{\partial x}(x)^{T} p+\frac{\partial\left(p^{T} R(x) p\right)^{T}}{\partial x}-\frac{\partial q^{T}}{\partial x} .
\end{array}\right.
$$

From the integrability assumption on (7) and the property of lifted Hamiltonian systems described in $\S \mathrm{IV}$, the Hamiltonian 
$H_{0}=p^{T} f(x)$ is integrable. We define the perturbation Hamiltonian by $H_{1}:=H-H_{0}=-\frac{1}{2} p^{T} R(x) p+q(x)$. The Hamiltonian $H_{1}$ is considered to represent the behaviors created by the control inputs, although this must be interpreted in the lifted space, the cotangent bundle $T^{*} M$.

We first solve the unperturbed Hamilton's canonical equations

$$
\dot{x}=f(x), \quad \dot{p}=-\frac{\partial f}{\partial x}(x)^{T} p
$$

determined by $H_{0}$ by means of the Hamilton-Jacobi theory. We take the Hamilton-Jacobi approach because it automatically produces new canonical variables. It is important to keep working with canonical variables not to cause secular terms in calculations, by which stability analysis may become unreliable (see, e.g., [12]).

The Hamilton-Jacobi equation to solve (9) is

$$
H_{0}\left(x, \frac{\partial W}{\partial x}\right)+\frac{\partial W}{\partial t}=0 .
$$

Proposition 2: A complete solution of (10) is obtained as $W(x, t, P)=\sum_{j=1}^{n} P_{j} \tilde{\Phi}_{j}(t, x), \quad P_{j}$ 's are arbitrary constants, where $\tilde{\Phi}(t, x)=\left(\tilde{\Phi}_{1}(t, x), \cdots, \tilde{\Phi}_{n}(t, x)\right)$ is the inverse of the solution $x=\Phi\left(t, x_{0}\right)$.

From $W(x, t, P)$, by

$$
p_{j}=\frac{\partial W}{\partial x}, \quad X_{j}=\frac{\partial W}{\partial P_{j}} \text { (arbitrary constants), }
$$

a general solution of (9) is obtained as

$$
x_{j}(t)=\Phi_{j}(t, X), \quad p_{j}(t)=\sum_{k=1}^{n} P_{k} \frac{\partial \tilde{\Phi}}{\partial x_{j}}(t, x)
$$

or,

$$
x(t, X)=\Phi(t, X), \quad p(t, X, P)=\frac{\partial \tilde{\Phi}}{\partial x}(t, x)^{T} P .
$$

We note that the transformation $(x, p) \rightarrow(X, P)$ is canonical. In the new coordinates the free motion (without control) is represented as

$$
\dot{X}=0, \quad \dot{P}=0 .
$$

With control, the new Hamiltonian is

$$
H_{1}(x, p)=H_{1}(x(t, X), p(t, X, P))=: \bar{H}_{1}(X, P, t),
$$

and $X, P$ obey

$$
\dot{X}_{j}=\frac{\partial \bar{H}_{1}}{\partial P_{j}}, \quad \dot{P}_{j}=-\frac{\partial \bar{H}_{1}}{\partial X_{j}} .
$$

We remark that until now no approximation has been made. If we plug the solution $X(t), P(t)$ of (13) into (11) or (12), we get exact solutions of the Hamilton's canonical equations (8) for the original control Hamilton-Jacobi equation (HJ). However, it is still difficult to solve (13) and we try to find an approximate solution of (13).
Assumption 2: The integrable vector field $f(x)$ is written as

$$
f(x)=A x+O\left(|x|^{2}\right), \quad A=\frac{\partial f}{\partial x}(0),
$$

where $A$ is a real $n \times n$ matrix. Furthermore, the linearization of (HJ), which is the algebraic Riccati equation

$$
P A+A^{T} P-P R(0) P+Q=0,
$$

has the stabilizing solution $\Gamma$.

We note that from Assumption 2, it follows that

$$
\begin{aligned}
& x=\Phi(t, X)=e^{A t} X+O\left(|X|^{2}\right) \\
& X=\tilde{\Phi}(t, x)=e^{-A t} x+O\left(|x|^{2}\right) .
\end{aligned}
$$

Proposition 3: The linearized equation of (13) is

$$
\left\{\begin{array}{l}
\dot{\bar{X}}=-e^{-A t} R(0) e^{-A^{T} t} \bar{P} \\
\dot{\bar{P}}=-e^{A^{T} t} Q e^{A t} \bar{X} .
\end{array}\right.
$$

Moreover, this can be explicitly solved as

$$
\begin{aligned}
&\left(\begin{array}{c}
\bar{X}\left(t, \bar{X}_{0}, \bar{P}_{0}\right) \\
\bar{P}\left(t, \bar{X}_{0}, \bar{P}_{0}\right)
\end{array}\right)=\left(\begin{array}{cc}
e^{A t} & 0 \\
0 & e^{-A^{T} t}
\end{array}\right)^{-1} \\
& \times \exp \left[t\left(\begin{array}{cc}
A & -R(0) \\
-Q & -A^{T}
\end{array}\right)\right]\left(\begin{array}{c}
\bar{X}_{0} \\
\bar{P}_{0}
\end{array}\right) .
\end{aligned}
$$

By Assumption 2, it follows that

$$
\left(\begin{array}{cc}
A & -R(0) \\
-Q & -A^{T}
\end{array}\right)\left(\begin{array}{l}
I \\
\Gamma
\end{array}\right)=\left(\begin{array}{l}
I \\
\Gamma
\end{array}\right)(A-R(0) \Gamma)
$$

and

$\exp \left[t\left(\begin{array}{cc}A & -R(0) \\ -Q & -A^{T}\end{array}\right)\right]\left(\begin{array}{l}I \\ \Gamma\end{array}\right)=\left(\begin{array}{l}I \\ \Gamma\end{array}\right) \exp [t(A-R(0) \Gamma)]$.

Therefore, if we take the initial conditions $\bar{X}_{0}$ and $\bar{P}_{0}$ satisfying $\bar{P}_{0}=\Gamma \bar{X}_{0}$, then, we have

$$
\left(\begin{array}{l}
\bar{X} \\
\bar{P}
\end{array}\right)=\left(\begin{array}{cc}
e^{-A t} & 0 \\
0 & e^{A^{T} t}
\end{array}\right)\left(\begin{array}{l}
I \\
\Gamma
\end{array}\right) \exp [t(A-R(0) \Gamma)] \bar{X}_{0} .
$$

Denoting quantities in the left-hand side of the above equation as $\bar{X}\left(t, \bar{X}_{0}, \Gamma \bar{X}_{0}\right)$ and $\bar{P}\left(t, \bar{X}_{0}, \Gamma \bar{X}_{0}\right)$, we substitute them in (12). Then, we have the following proposition.

Proposition 4: For sufficiently small $\left|\bar{X}_{0}\right|$,

$$
x\left(t, \bar{X}\left(t, \bar{X}_{0}, \Gamma \bar{X}_{0}\right)\right), \quad p\left(t, \bar{X}\left(t, \bar{X}_{0}, \Gamma \bar{X}_{0}\right), \bar{P}\left(t, \bar{X}_{0}, \Gamma \bar{X}_{0}\right)\right)
$$

converge to the origin as $t \rightarrow \infty$.

From Proposition 4, we think of

$$
x\left(t, \bar{X}\left(t, \bar{X}_{0}, \Gamma \bar{X}_{0}\right)\right), p\left(t, \bar{X}\left(t, \bar{X}_{0}, \Gamma \bar{X}_{0}\right), \bar{P}\left(t, \bar{X}_{0}, \Gamma \bar{X}_{0}\right)\right)
$$

as approximated behaviors of the canonical equations (8) near the stable Lagrangian submanifold. By changing $t$ and $\bar{X}_{0}$ the points defined by (19) move on a surface which will be an approximation of the stable Lagrangian submanifold. Actually, one direction in $\bar{X}_{0}$ space is not independent of $t$. Therefore, we eliminate $t$ and $n-1$ elements of $\bar{X}_{0}=\left(\bar{X}_{01}, \ldots, \bar{X}_{0 n}\right)$ to get an approximation of the stable Lagrangian submanifold. Summarizing, we propose 
the following method to approximate the stable Lagrangian submanifold and the stabilizing feedback.

Proposition 5: Solve the uncontrolled system equation $\dot{x}=f(x)$ of (7). Form a general solution (11) or (12) of (9) using the solution of $\dot{x}=f(x)$. Calculate $\bar{X}\left(t, \bar{X}_{0}, \bar{P}_{0}\right)$ and $\bar{P}\left(t, \bar{X}_{0}, \bar{P}_{0}\right)$ in (18) and restrict their initial points to the stable Lagrangian subspace of the linearized Riccati equation around the origin, $\left\{\left(\bar{X}_{0}, \Gamma \bar{X}_{0}\right)|| \bar{X}_{0} \mid\right.$ small $\}$, where $\Gamma$ is the stabilizing solution. Substitute them in (11) (or (12)) to get (19). Eliminate $t$ and $n-1$ variables from $\bar{X}_{01}, \ldots, \bar{X}_{0 n}$ to get a set of $n$ algebraic equations of $x_{1}, \ldots, x_{n}$ and $p_{1}, \ldots, p_{n}$, which defines an $n$-dimensional surface. Solve the algebraic equation with respect to $p_{1}, \ldots, p_{n}$ to get a graph form expression of the surface $p=p(x)$ (or, $\left.p_{1}=p_{1}(x), \ldots, p_{n}=p_{n}(x)\right)$. One can construct the desired feedback from $p(x)$ depending on $R$ in (HJ).

Based on the next proposition, one can expect that the performance of the feedback control in Proposition 5 is better than that of the linear control obtained from (14).

Proposition 6: If the procedure in Proposition 5 is applied to the linearized system and the Riccati equation (14), the resulting solution is the linear stabilizing solution $\Gamma$.

\section{NUMERICAL EXAMPLE}

Let us consider the 1-dimensional nonlinear optimal control problem;

$$
\begin{aligned}
& \dot{x}=x-x^{3}+u \\
& J=\int_{0}^{\infty} \frac{q}{2} x^{2}+\frac{r}{2} u^{2} d t .
\end{aligned}
$$

The Hamilton-Jacobi equation for this problem is

$$
H=p\left(x-x^{3}\right)-\frac{1}{2 r} p^{2}+\frac{q}{2} x^{2}=0
$$

and the Hamilton's canonical equations are

$$
\left\{\begin{array}{l}
\dot{x}=x-x^{3}-\frac{1}{r} p \\
\dot{p}=-\left(1-3 x^{2}\right) p-q x .
\end{array}\right.
$$

The Hamiltonian $H$ is split into the integrable and perturbation parts;

$$
H_{0}=p\left(x-x^{3}\right), \quad H_{1}=-\frac{1}{2 r} p^{2}+\frac{q}{2} x^{2} .
$$

The solution of the equation (20) with the initial condition $x=X$ at $t=0$ without control is obtained from

$$
\frac{x^{2}}{1-x^{2}}=\frac{X^{2}}{1-X^{2}} e^{2 t},
$$

and is denoted as $x=\Phi(t, X)$. The solution of the canonical equations for $H_{0}$ corresponding to (9) is

$$
x=\Phi(t, X), \quad p=D \tilde{\Phi}(t, x) P=\frac{X^{3} e^{2 t}}{x^{3}} P,
$$

where $\tilde{\Phi}$ is the inverse of $\Phi$ with respect to $x, P$ is an arbitrary constant and the last equation is derived from (22).
Based on the linearization of (20) $(A=1, B=1)$, the linearized canonical equations for perturbation that corresponds to (9) are

$$
\left\{\begin{array}{l}
\dot{\bar{X}}=-\frac{1}{r} e^{-2 t} \bar{P} \\
\dot{\bar{P}}=-q e^{2 t} \bar{X} .
\end{array}\right.
$$

The solution of the above equations for the initial condition in the stable Lagrangian subspace of the linearized Riccati equation of (21) is

$$
\left\{\begin{array}{l}
\bar{X}\left(t, \bar{X}_{0}, \Gamma \bar{X}_{0}\right)=e^{-(1+\lambda) t} \bar{X}_{0} \\
\bar{P}\left(t, \bar{X}_{0}, \Gamma \bar{X}_{0}\right)=e^{(1-\lambda) t} \Gamma \bar{X}_{0},
\end{array}\right.
$$

where $\Gamma=r+\sqrt{r^{2}+q r}$ is the stabilizing solution of the Riccati equation and $-\lambda=-\sqrt{1+q / r}$ is the closed loop matrix (eigenvalue). Substituting the expression of $\bar{X}$ into the first equation of (23), we have

$$
\frac{x^{2}}{1-x^{2}}=\frac{e^{-2 \lambda t} \bar{X}_{0}^{2}}{1-e^{-2(1+\lambda) t} \bar{X}_{0}^{2}},
$$

from which we write down $t$ as a function of $x$. Next, we substitute the expressions of $t(x)$ just obtained and $\bar{X}$ and $\bar{P}$ into the second equation of (23). Then, we have an approximation of the asymptotically stable Lagrangian submanifold in the graph form

$$
p=p(x),
$$

and $u=-(1 / r) p(x)$ is an approximation of the optimal feedback control.

\section{Remarks.}

(i) When $q / r \gg 1$, in other words, when a high gain feedback is allowed, by approximating $e^{-2 \lambda t} \sim e^{-2(1+\lambda) t}$, (23) gives $x^{2}=e^{-2 \lambda t} \bar{X}_{0}^{2}$. Thus, we have $p(x)=\Gamma x$, which is the linear optimal feedback.

(ii) When $t \gg 1$ in (23), it follows that

$$
\frac{e^{-2 \lambda t} \bar{X}_{0}^{2}}{1-e^{-2(1+\lambda) t} \bar{X}_{0}^{2}} \sim e^{-2 \lambda t} \bar{X}_{0}^{2}, \quad \frac{x^{2}}{1-x^{2}} \sim x^{2}
$$

and hence $e^{-2 \lambda t} \bar{X}_{0}^{2} \sim x^{2}$. Thus, it can be seen that the feedback (26) is tangent to the linear optimal feedback at the origin (see, also Proposition 6);

$$
p(x) \sim \Gamma x .
$$

Calculations have been carried out with $q=1$ and $r=1$. The important step of the calculations is to solve (25) with respect to $t$. We have done it for sampled points of $x$ in $[0,2]$ with step size 0.05 . The values of $t$ are substituted, first in (24) which gives a relation of $x$ and $\bar{X}$ and, next in (23) together with the $x-X$ relation, yielding a relation of $p$ and $x$ at the sample points. Finally, the $p-x$ relation thus obtained is interpolated with a spline function. The result is shown in Fig. 1, which is an approximation of the optimal feedback.

One knows that the Hamilton-Jacobi equation (21) can be solved exactly since the problem is one dimensional. Actually, we are more concerned with (stabilizing) feedback function rather than solutions of (21). It is easily obtained 


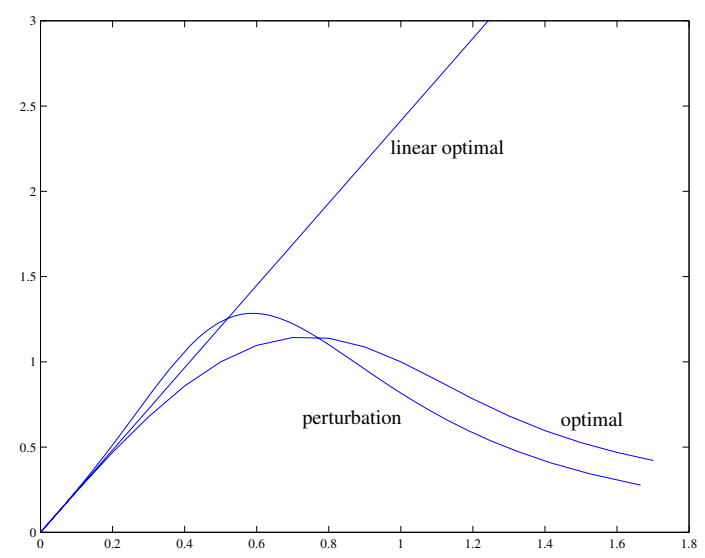

Fig. 1. Sub-optimal (pertrubation), linear optimal, and optimal feedback.

by solving the quadratic equation for $p$ and taking a positive solution. Fig. 1 shows the optimal feedback showing a resemblance to the sub-optimal feedback.

\section{CONCLUDING REMARKS AND FUTURE WORK}

In this report we have proposed an approximation method for the stabilizing solution of the Hamilton-Jacobi equation for integrable systems. The approach in this research has several unique features. First, we have taken full account of the geometric studies done by the authors using symplectic geometry. Second, techniques from Hamiltonian mechanics are used, which is quite natural since the well-established theory for the Riccati equation is based on the Hamiltonian matrix. Third, we have shown that the knowledge on the system without control, which is the complete expression of general solutions, can be effectively used for controller design when the framework is properly chosen.

As is shown in the numerical example, the quantitative error of the obtained feedback function from the optimal one is apparent. To eliminate this, calculations following the linearization of (13) need to be considered. Also, for higher dimensional systems, efficient software use to solve the algebraic equation defining the approximated Lagrangian submanifold is necessary although the theory presented itself can be applied for any finite dimensional system.

Acknowledgement. The first author was supported by the Scientist Exchange Program between the Japan Society for the Promotion of Science (JSPS) and the Netherlands Organisation for Scientific Research (NWO).

\section{REFERENCES}

[1] R. Abraham and J. E. Marsden. Foundations of Mechanics. AddisonWesley, New York, 2nd edition, 1979.

[2] V. I. Arnold. Mathematical Methods of Classical Mechanics. SpringerVerlag, 2nd edition, 1989.

[3] J. A. Ball, J. W. Helton, and M. L. Walker. $H_{\infty}$ control for nonlinear systems with output feedback. IEEE Trans. Automat. Control, 38(4):546-559, 1993.

[4] J. A. Ball, M. A. Petersen, and A. van der Schaft. Inner-outer factorization for nonlinear noninvertible systems. IEEE Trans. Automat. Control, 49(4):483-492, 2004.
[5] J. A. Ball and A. J. van der Schaft. $J$-inner-outer factorization, $J$ spectral factorization, and robust control for nonlinear systems. IEEE Trans. Automat. Control, 41(3):379-392, 1996.

[6] M. Bardi and I. Capuzzo-Dolcetta. Optimal Control and Viscosity Solutions of Hamilton-Jacobi-Bellman Equations. Birkhauser, 1997.

[7] R. W. Beard, G. N. Sardis, and J. T. Wen. Galerkin approximations of the generalized Hamilton-Jacobi-Bellman equation. Automatica, 33(12):2195-2177, 1997.

[8] M. G. Crandall and P. L. Lions. Viscosity solutions of Hamilton-Jacobi equations. Trans. AMS, 277:1-43, 1983.

[9] P. E. Crouch and A. J. van der Schaft. Variational and Hamiltonian Control Systems. Springer-Verlag, 1987.

[10] W. H. Fleming and H. M. Soner. Controlled Markov Processes and Viscosity Solutions. Springer, 2nd edition, 2005.

[11] K. Fujimoto and J. M. A. Scherpen. Nonlinear input-normal realizations based on the differential eigenstructure of Hankel operators. IEEE Trans. Automat. Control, 50(1):2-18, 2005.

[12] H. Goldstein. Classical Mechanics. Addison-Wesley, 3rd edition, 2001.

[13] D. Hill and P. Moylan. The stability of nonlinear dissipative systems. IEEE Trans. Automat. Control, 21(10):708-711, 1976.

[14] A. Isidori and A. Astolfi. Disturbance attenuation and $H_{\infty}$-control via measurement feedback in nonlinear systems. IEEE Trans. Automat. Control, 37(9):1283-1293, 1992.

[15] E. B. Lee and L. Markus. Foundations of Optimal Control Theory. John Wiley, New York, 1967.

[16] D. L. Lukes. Optimal regulation of nonlinear dynamical systems. SIAM J. Control Optim., 7(1):75-100, 1969.

[17] C. P. Mracek and J. R. Cloutier. Control designs for the nonlinear benchmark problem via the state-dependent Riccati equation method. Int. J. Robust and Nonlinear Control, 8:401-433, 1998.

[18] T. Oshima and H. Komatsu. Partial Differential Equations of the First Order. Iwanami Shoten, Tokyo, 1977. (in Japanese).

[19] N. Sakamoto. Analysis of the Hamilton-Jacobi equation in nonlinear control theory by symplectic geometry. SIAM J. Control Optim., 40(6): 1924-1937, 2002.

[20] P. Soravia. $H^{\infty}$ control of nonlinear systems: Differential games and viscosity solutions. SIAM J. Control Optim., 34(3):1071-1097, 1996.

[21] A. J. van der Schaft. On a state space approach to nonlinear $H_{\infty}$ control. Syst. Control Lett., 16(1):1-18, 1991.

[22] A. J. van der Schaft. $L_{2}$-gain analysis of nonlinear systems and nonlinear state feedback $H_{\infty}$ control. IEEE Trans. Automat. Control, 37(6):770-784, 1992.

[23] A. J. van der Schaft. L2-Gain and Passivity Techniques in Nonlinear Control. Springer, 2nd edition, 1999.

[24] J. C. Willems. Dissipative dynamical systems-Part I, II. Arch. Rational Mechanics and Analysis, 45:321-393, 1972. 